\title{
IMPROVED MUCKENHOUPT-WHEEDEN INEQUALITY AND \\ WEIGHTED INEQUALITIES FOR POTENTIAL OPERATORS
}

\author{
Y. RAKOTONDRATSIMBA
}

\begin{abstract}
By a variant of the standard good $\lambda$ inequality, we prove the Muckenhoupt-Wheeden inequality for measures which are not necessarily in the Muckenhoupt class. Moreover we can deal with a general potential operator, and consequently we obtain a suitable approach to the two weight inequality for such an operator when one of the weight functions satisfies a reverse doubling condition.
\end{abstract}

\section{Introduction}

In this paper $d \mu, d \omega$ are locally finite positive Borel measures of $R^{n}$, $n \geq 1$. For a nonnegative locally- $d \mu$ integrable function $K(x, y)$ (a.e. continuous in the first variable) we define the potential operator

$$
(T f \mu)(x)=\int_{y \in \mathbb{R}^{n}} K(x, y) f(y) d \mu(y) .
$$

For each $C_{1}>0$, we assume the existence of a $C_{2}>0$ so that

$(\mathcal{H}) K(x, y) \leq C_{2} K(z, y)$, for each $x, y, z$ with $0<|z-y|<C_{1}|x-y|$.

The dual operator $T^{*}$ is the operator defined by the kernel $K^{*}(x, y)=$ $K(y, z)$. The usual fractional integral operator $I_{s}$, with $0<s<n$, is given by $K(x, y)=|x-y|^{s-n}$. Other examples of operators $T$ are those introduced by Chanillo-Stromberg-Wheeden [Ch-St-Wh] and given by kernels $K(x, y)=\frac{a(y,|x-y|)}{|x-y|^{n}}$. Here $a$ is considered as a function defined on balls of $\mathbb{R}^{n}$ and which satisfies some growth conditions we precise below. 
We are interested in finding a constant $C>0$ for which

$\left(P_{T}\right) \quad\|T f \mu\|_{L_{\omega}^{q}} \leq C\|f\|_{L_{\mu}^{p}}$ for all nonnegative functions $f$

with $1<p, q<\infty$. The constant $C$ depends only on $n, p, q, \omega, \mu$ and $K$; and when it is necessary we denote this dependance by writing $C=$ $C(n, p, q, K, \omega, \mu)$. Here $\|g\|_{L_{\nu}^{r}}=\left(\int_{\mathbb{R}^{n}}|g|^{r} d \nu\right)^{\frac{1}{r}}$. The inequality $\left(P_{T}\right)$ includes the usual two weight norm inequality

$$
\|T f\|_{L_{u}^{q}} \leq C\|f\|_{L_{v}^{p}}
$$

since it is sufficient to replace $f$ by $f v^{\frac{1}{p-1}}$, and to take $d \omega=u d x, d \mu=$ $v^{-\frac{1}{p-1}} d x$, where $d x$ is the usual Lebesgue measure on $\mathbb{R}^{n}$. Inequality $\left(P_{T}\right)$ with $T=I_{s}$ has been studied extensively by many authors (see for instance $[\mathbf{K e}-\mathbf{S a}],[\mathbf{S a}-\mathbf{W h}]$ and $[\mathbf{P e}]$ and the reference given by them). Kerman and Sawyer $[\mathbf{K e - S a}]$ solved the problem $\left(P_{I_{s}}\right)$ with $d \omega=d x$. This particular case is first interesting since it is the usual form which appears in many mathematic and physic areas. It also appears that the case $d \omega=d x$ is naturally suitable to be treated. In fact using a good $\lambda$-inequality, they proved that the left member of $\left(P_{T}\right)$ is majorized by the $L^{q}$ norm of the fractional maximal function. So $\left(P_{T}\right)$ is reduced to a weighted inequality for maximal operator, whose study was done by the first author $[\mathbf{S a}]$. Problem $\left(P_{T}\right)$ with general measures $d \mu$ and $d \omega$ was solved by Sawyer and Wheeden [Sa-Wh].

Let us consider the operator $T=I_{s}$ with $0<s<n$. We have the pointwise inequality

$$
M_{s} g \leq c(s, n) I_{s} g
$$

where $M_{s}$ is the fractional maximal operator defined by

$$
\left(M_{s} f\right)(x)=\sup \left\{|Q|^{\frac{s}{n}-1}\left\|f \mathbb{1}_{Q}\right\|_{L^{1}(d y)} ; Q \text { a cube with } Q \ni x\right\} .
$$

We generally use the letter $Q$ to denote a cube of $\mathbb{R}^{n}$, and by which we mean a product of $n$ intervals $\left[a_{i}, a_{i}+t\right](0<t<\infty)$. The MuckenhouptWheeden inequality $[\mathbf{M u}-\mathbf{W h}]$ yields a sort of converse (in norm) of the above inequality, and asserts that for $0<q<\infty$ :

$$
\left\|I_{s} f\right\|_{L_{\omega}^{q}} \leq c(s, n, q, \omega)\left\|M_{s} f\right\|_{L_{\omega}^{q}} \text { for all functions } f
$$

whenever the measure $d \omega$ satisfies the Muckenhoupt condition $A_{\infty}$, i.e. there are $c=c(\omega), \delta>0$ such as

$$
\frac{|E|_{\omega}}{|Q|_{\omega}} \leq c\left(\frac{|E|}{|Q|}\right)^{\delta} \text { for all cubes } Q \text { and all measurables sets } E \subset Q \text {. }
$$


In his thesis Perez $[\mathbf{P e}]$ gave a weaker condition than the $A_{\infty}$ 's. He proved the above Muckenhoupt-Wheeden inequality for measures $d \omega$ satisfying $D_{\infty}$ and $B_{\rho}$ conditions with $\rho>1-\frac{s}{n}$, and which can be noted as $d \omega \in D_{\infty} \cap B_{\rho}$. These conditions respectively mean:

$$
|2 Q|_{\omega}=\int_{2 Q} \omega \leq C(\omega)|Q|_{\omega} \text { for all cubes } Q,
$$

(here $2 Q$ is the cube having the same center as $Q$ and the length expanded twice)

$$
\frac{\left|Q^{\prime}\right| \omega}{|Q|_{\omega}} \leq C^{\prime}(\omega)\left(\frac{\left|Q^{\prime}\right|}{|Q|}\right)^{\rho} \text { for all cubes } Q, Q^{\prime} \text { with } Q^{\prime} \subset Q .
$$

Contrary to the Muckenhoupt-Wheeden technique $[\mathbf{M u}-\mathbf{W h}]$, the Perez's analysis $[\mathbf{P e}]$ is not based on the standard good- $\lambda$ inequalities. This last author used some estimates obtained by Frazier and Jawerth [Fr-Ja] for local maximal operator, and moreover he was able to treat the problem with a general convolution operator.

In this paper we also prove the Muckenhoupt-Wheeden inequality for measures which are not necessarily in the Muckenhoupt class (see Corollary 4), and with the general potential operator $T$ described above. We do this, with a sort of a variant of the standard good $\lambda$ inequality and by introducing a suitable maximal operator $M_{T, \omega}$ (see Theorem 1). The additional conditions on the measure $d \omega$ arise only in order to relate this "exotic" maximal operator to a more standard one like $M_{s}$ (see Theorem 3). Consequently we obtain a suitable approach to the two weight inequality for such an operator when one of the weight functions satisfies a reverse doubling condition (see Theorem 5).

\section{Statements of results}

Let us define the dyadic maximal operator

$$
\begin{gathered}
\left(M_{T, \omega, \mu}^{d} f\right)(x)=\sup \left\{|Q|_{\omega}{ }^{-1}\left\|f\left(T^{*} \mathbb{I}_{Q} \omega\right) \mathbb{I}_{3 Q}\right\|_{L^{1}(d \mu)} ;\right. \\
Q \text { a dyadic cube with } Q \ni x\} .
\end{gathered}
$$

A dyadic cube $Q$ is a product of $n$ intervals of the form $\left[2^{k} a_{i}, 2^{k}\left(a_{i}+1\right)\right]$, where $k$ and $a_{i}$ are integers. Fix $q \geq 1$. By using the Holder inequality we can observe that $\left(M_{T, \omega, \mu}^{d} f\right)(x)$ is a.e. finite for all bounded functions with compact supports whenever measures $d \omega$ and $d \mu$ satisfy the condition

$$
\left\|T \mathbb{I}_{|x|<R} \mu\right\|_{L_{\omega}^{q}} \leq c(R)<\infty \text { for all } R>0 .
$$

Our first result is as follow: 


\section{Theorem 1.}

Let $0<q<\infty$ and $K$ be a nonnegative kernel satisfying the hypothesis $\mathcal{H}$. Assume the measures $d \omega$ and $d \mu$ satisfy the condition $\left(S_{T}\right)$. Then there is $C=C(n, q, K)>0$ so that

$$
\|T f \mu\|_{L_{\omega}^{q}} \leq C\left\|M_{T, \omega, \mu}^{d} f\right\|_{L_{\omega}^{q}} .
$$

If $M_{\omega}^{d}$ is the dyadic maximal operator defined by

$$
\left(M_{\omega}^{d} f\right)(x)=\sup \left\{|Q|_{\omega}^{-1}\left\|f \mathbb{I}_{Q}\right\|_{L^{1}(d \omega)} ; Q \text { a dyadic cube with } Q \ni x\right\},
$$

then (see Lemma 1 below)

$$
\left(M_{T, \omega, \mu}^{d} g\right)(.) \leq\left(M_{\omega}^{d}(T g \mu)\right)(.)
$$

and consequently we get

\section{Proposition 2.}

Let $K, d \omega, d \mu$ be as above Then for $q>1$ we have

$$
\|T f \mu\|_{L_{\omega}^{q}} \approx\left\|M_{T, \omega, \mu}^{d} f \mu\right\|_{L_{\omega}^{q}} .
$$

Moreover this equivalence also holds for the range of $q \in] 0,1]$ whenever

$$
M_{\omega}^{d}(T g)(.) \leq c(n, K, \omega)(T g)(.)
$$

for all $f$ nonnegative functions $g$.

The above equivalence means

$$
C_{1}(n, q, K)\|T f \mu\|_{L_{\omega}^{q}} \leq\left\|M_{T, \omega, \mu}^{d} f \mu\right\|_{L_{\omega}^{q}} \leq C_{2}(n, q, \omega)\|T f \mu\|_{L_{\omega}^{q} .}
$$

The extra assumption in this result is satisfied for instance for the kernel $K(x, y)=|x-y|^{s-n}$, with $d \omega=d x$ the Lebesgue measure, and more generally for measures $d \omega \in D_{\infty} \cap B_{\rho}$ with $1-\frac{s}{n}<\rho$.

Thus in view of Theorem 1 , the inequality $\left(P_{T}\right)$ is reduced to the following one, related for $M_{T, \omega, \mu}^{d}$

$$
\left\|M_{T, \omega, \mu}^{d} f \mu\right\|_{L_{\omega}^{q}} \leq C\|f\|_{L_{\mu}^{p}}
$$

In order to get this last one, we impose more hypothesis on the kernel $K$. So as to simplify, we only deal with kernels

$$
K(x, y)=K_{a}(x, y)=\frac{a(y,|x-y|)}{|x-y|^{n}}=\frac{a(B(y,|x-y|))}{|x-y|^{n}}
$$


where $a$ is a function defined on balls satisfying the following hypotheses $H$ :

(i) $\quad a\left(B_{1}\right) \leq c_{1}(n, a) a\left(B_{2}\right)$ for all balls $B_{1}, B_{2}$ with $B_{1} \subset B_{2}$;

there are $\lambda, \sigma>0$ so that

(ii) $c_{1}^{\prime}(n, a) t^{n \lambda} a(B) \leq a(t B) \leq c_{2}^{\prime}(n, a) t^{n \sigma} a(B)$ for all balls $B$ and $t \geq 1$.

We also define the function $a$ on cubes by $a(Q)=a(B)$, where $B$ is the smallest ball which contains the cube $Q$. A suitable dyadic maximal operator related to the potential operator $T=T_{a}$ (with kernel $K=K_{a}$ ) is

$\left(M_{\Phi}^{d} f\right)(x)=\sup \left\{a(Q)|Q|^{-1}\left\|f \mathbb{I}_{Q}\right\|_{L^{1}(d y)} ; Q\right.$ a dyadic cube with $\left.Q \ni x\right\}$.

The nondyadic version of $M_{\Phi}^{d}$ is merely denoted by $M_{\Phi}$. The measure $d \omega$ satisfies the condition $R D_{\rho}$ with $\rho>0$ (and we write as $d \omega \in R D_{\omega}$ ) when there $c=c(\omega, n)>0$ for which

$$
t^{n \rho}|B|_{\omega} \leq c|t B|_{\omega} \text { for all balls } B \text { and } t>1
$$

Our second result ensures the link between the two maximal operators we have defined above.

\section{Theorem 3.}

Let $K=K_{a}$ be a kernel satisfying $\mathcal{H}$ i)-ii) with $0<\lambda, \sigma \leq 1$. Suppose $d \omega \in R D_{\rho}$ with $1-\lambda<\rho$. Then

$$
C_{1}\left(M_{\Phi}^{d} f \mu\right)(.) \leq\left(M_{T_{a}, \omega, \mu}^{d} f\right)(.) \leq C_{2}\left(M_{\Phi} f \mu\right)(.) \text { for all functions } f
$$

here $C_{1}=C_{1}(n, a)>0$ and $C_{2}=C_{2}(n, a, \omega)$.

In fact $C_{2}$ does not depend on the individual measure $d \omega$ but only on the $R D_{\rho}$ constant of $d \omega$. The claim we announced in the introduction can be stated as

\section{Corollary 4.}

Let $0<s<n$ and $0<q<\infty$. Suppose $d \omega \in R D_{\omega}$ with $\left(1-\frac{s}{n}\right)<\rho$. Then

$$
\left\|I_{s} g\right\|_{L_{\omega}^{q}} \approx\left\|M_{s} g\right\|_{L_{\omega}^{q}} \text { for all nonnegative functions } g
$$

whenever

$$
\int_{R<|x|}|x|^{(s-n) q} d \omega(x)<c(R)<\infty \text { for all } R>0 .
$$


This is an immediate consequence of Theorems 1 and 2. Indeed since for all $R>0$ :

$\left(I_{s} \mathbb{1}_{B(0, R)}\right)(.) \mathbb{1}_{B(0,2 R)}(.) \approx R^{s} \mathbb{I}_{B(0,2 R)}($.$) and \left(I_{s} \mathbb{I}_{B(0, R)}\right)(x) \mathbb{I}_{|x|>2 R}(x) \approx$ $|x|^{s-n} \mathbb{I}_{|x|>2 R}(x)$ so the condition $\left\|I_{s} \mathbb{I}_{B(0, R)}\right\|_{L_{\omega}^{q}}<\infty$ is reduced to the one written in this corollary. Note also in studying the two weight inequality $\left\|I_{s} f\right\|_{L_{\omega}^{q}} \leq c\|f\|_{L_{\nu}^{q}}$ it is necessary that $\left\|I_{s} \mathbb{I}_{B(0, R)}\right\|_{L_{\omega}^{q}} \leq c\left\|\mathbb{I}_{B(0, R)}\right\|_{L_{\nu}^{p}}<\infty$.

By Theorems 1 and 3 , the problem $\left(P_{T}\right)$ is then reduced to the following maximal inequality

$$
\left\|M_{\Phi} f \mu\right\|_{L_{\omega}^{q}} \leq c\|f\|_{L_{\mu}^{p}}
$$

By the study of this last case (see [Ra1], or adapt the proof given in $[\mathbf{S a}])$ then we get

\section{Theorem 5.}

Let $1<p, q<\infty$, and $K=K_{a}$ be a kernel satisfying $\mathcal{H} i$ )-ii) with $0<\lambda, \sigma<1$. Suppose $d \omega \in R D_{\omega}$ with $(1-\lambda)<\rho$. Then the inequality

$$
\|T f \mu\|_{L_{\omega}^{q}} \leq c\|f\|_{L_{\mu}^{p}}
$$

holds if and only if

$$
\int_{|x|>R}\left[\frac{a(x,|x|)}{|x|^{n}}\right]^{q} d \omega(x)<c(R)<\infty \text { for all } R>0,
$$

and

$$
\left\|\left(T \sum_{k} \varepsilon_{k} \mathbb{I}_{Q_{k}} \mu\right) \mathbb{I}_{\bigcup} Q_{k}\right\|_{L_{\omega}^{q}} \leq C\left\|\sum_{k} \varepsilon_{k} \mathbb{I}_{Q_{k}}\right\|_{L_{\mu}^{p}},
$$

where $C>0$ is a constant which does not depend of each sequence $\left(Q_{k}\right)_{k}$ of cubes and $\left(\varepsilon_{k}\right)_{k}$ of nonnegative reals $\varepsilon_{k}$.

Moreover in the case $1<p \leq q$ the condition (2) can be replaced by

$$
\left\|\left(T \mathbb{1}_{Q} \mu\right) \mathbb{1}_{Q}\right\|_{L_{\omega}^{q}} \leq C\left\|\mathbb{1}_{Q}\right\|_{L_{\mu}^{p}} \text { for all cubes } Q
$$

Sawyer and Wheeden $[\mathbf{S a}-\mathbf{W h}]$ proved that for $1<p \leq q$ and for all general measures $d \omega$ and $d \mu$, then $\left(P_{T}\right)$ is equivalent to $\left(2^{\prime}\right)$ and

$$
\left\|\left(T^{*} \mathbb{I}_{Q} \omega\right) \mathbb{I}_{Q}\right\|_{L_{\mu}^{p^{\prime}}} \leq c\left\|\mathbb{I}_{Q}\right\|_{L_{\omega}^{q^{\prime}}} \quad p^{\prime}=\frac{p}{p-1}, q^{\prime}=\frac{q}{q-1} .
$$

With an additional hypothesis on the measure $d \mu$ we can simplify the conditions in Theorem 5. We first consider the case $p \leq q$. 


\section{Proposition 6.}

Let $1<p \leq q<\infty$, and $K=K_{a}$ be a kernel satisfying $\mathcal{H}$ i)-ii) with $0<\lambda, \sigma<1$. Suppose $d \omega \in R D_{\rho}, d \mu \in R D_{\rho^{\prime}}$ with $(1-\lambda)<\rho$ and $\rho^{\prime}>0$. Then the inequality $\left(P_{T}\right)$ holds if and only if

$$
\left\|\left(T \mathbb{1}_{Q} \mu\right) \mathbb{1}_{Q}\right\|_{L_{\omega}^{q}} \leq C\left\|\mathbb{1}_{Q}\right\|_{L_{\mu}^{p}} .
$$

If moreover $d \mu \in R D_{\rho^{\prime}}$ with $(1-\lambda)<\rho^{\prime}$ or $d \mu \in A_{\infty}$ then, an easy necessary and sufficient condition for $\left(P_{T}\right)$ is

$$
\frac{a(Q)}{|Q|}|Q|_{\mu}^{1-\frac{1}{p}}|Q|_{\omega}^{\frac{1}{q}} \leq C \text { for all cubes } Q .
$$

This second part is already known $[\mathbf{S a}-\mathbf{W h}]$, and here we deduce it by using results on maximal functions (see $[\mathbf{R a 2}]$ and $[\mathbf{P e}]$ ). To deal with the range of $q<p$, we introduce the two conditions $d \mu \in \widetilde{R D}(p), d \omega \in D_{\varepsilon, q}$ with $\varepsilon \in[1, \infty[$ (see [Ch-St-Wh] $)$ and which mean respectively

$$
\begin{gathered}
\left\|\sum_{j \geq 0} \sum_{k} \varepsilon_{k}\left(\frac{\left|Q_{k}\right|_{\mu}}{\left|2^{j} Q_{k}\right|_{\mu}}\right) \mathbb{I}_{2^{j} Q_{k}}\right\|_{L_{\mu}^{p}} \leq c(\mu)\left\|\sum_{k} \varepsilon_{k} \mathbb{I}_{Q_{k}}\right\|_{L_{\mu}^{p}} \\
\left\|\sum_{k} \varepsilon_{k} \mathbb{I}_{t Q_{k}}\right\|_{L_{\omega}^{q}} \leq c(\omega) t^{n \varepsilon \frac{1}{q}}\left\|\sum_{k} \varepsilon_{k} \mathbb{I}_{Q_{k}}\right\|_{L_{\omega}^{q}}
\end{gathered}
$$

for all $t \geq 1, \varepsilon_{k}>0$ and all cubes $Q$ and $Q_{k}$. Thus we can state

\section{Proposition 7.}

Let $1<q<p<\infty$, and $K=K_{a}$ be a kernel satisfying $\mathcal{H}$ i)-ii) with $0<\lambda, \sigma<1$, and $d \omega \in R D_{\rho}$, with $(1-\lambda)<\rho$.

Suppose $d \mu \in \widetilde{R D}(p)$. Then the inequality $\left(P_{T}\right)$ holds if and only if for some $m \geq 4$ and $C>0$

$$
\left\|\sum_{k} \varepsilon_{k}\left(T \mathbb{I}_{Q_{k}}\right) \mathbb{I}_{\left(m Q_{k}\right)}\right\|_{L_{\omega}^{q}} \leq C\left\|\sum_{k} \varepsilon_{k} \mathbb{I}_{Q_{k}}\right\|_{L_{\mu}^{p}}
$$

for all cubes $Q_{k}$ and all $\varepsilon_{k}>0$.

For $d \mu \in R D_{\rho^{\prime}} \cap D_{\varepsilon^{\prime}, p}$ with $\max \left(1-\lambda, \frac{1}{p} \varepsilon^{\prime}\right)<\rho^{\prime}$, a necessary and sufficient condition for $\left(P_{T}\right)$ is

$$
\left\|\sum_{k} \varepsilon_{k}\left(\frac{a\left(Q_{k}\right)}{\left|Q_{k}\right|}\left|Q_{k}\right|_{\mu}\right) \mathbb{1}_{Q_{k}}\right\|_{L_{\omega}^{q}} \leq C\left\|\sum_{k} \varepsilon_{k} \mathbb{I}_{Q_{k}}\right\|_{L_{\mu}^{p}} .
$$


This equivalence is also true when $d \mu \in R D_{\rho} \cap D_{\infty}$, dw $\in D_{\varepsilon, q}$ with $1-\lambda<\rho^{\prime}$ and $\varepsilon<q(1-\sigma)$.

Remark. Now we show that the use of the sharp maximal $M^{\#}$ (see [Ya] for a definition) is not well adapted to weaken the weight condition in the Muckenhoupt-Wheeden inequality

$$
\left\|I_{s} f\right\|_{L_{\omega}^{q}} \leq c\left\|M_{s} f\right\|_{L_{\omega}^{q}}
$$

Indeed such a purpose is based on the two inequalities:

$$
\left(I_{s} f\right)^{\#} \leq c\left(M_{s} f\right)
$$

$$
\|g\|_{L_{\omega}^{q}} \leq c\left\|g^{\#}\right\|_{L_{\omega}^{q}}
$$

Inequality (2) is valid for all functions $f$ with $\left(I_{s} f\right) \in L_{\text {loc }}^{1}$ and was proved in $[\mathbf{A d}]$. Although (3) is well known to be true for $w \in A_{\infty}$, Yabuta [Ya] had obtained such an inequality with a weak condition he denoted as $w \in C_{r}$ (with $r>q$ ). Thus we think get (1) with this last condition. But since $M_{s} f \leq c I_{s} f=h$ then

$$
\left\|h^{\#}\right\|_{L_{\omega}^{q}} \leq c\|h\|_{L_{\omega}^{q}}
$$

It was proved in $[\mathbf{Y a}]$ that condition like (4) implies necessarily $w \in A_{\infty}$.

\section{Some Lemmas}

We first state two Lemmas we need and then we give their proofs.

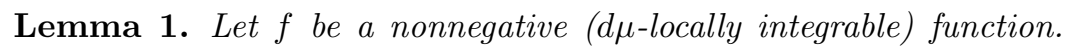
Then

$$
\left(M_{T, \omega, \mu}^{d} f\right)(.) \leq\left(M_{\omega}^{d}(T f \mu)\right)(.) .
$$

Lemma 2. Let $T=T_{a}$ be an operator with the kernel $K=K_{a}$ satisfying $\mathcal{H}$ i)-ii), and let $d \nu$ be a positive Borel measure.

A) If $0<\sigma \leq 1$ then there is $C=C(a, n)>0$ so that for all cubes $Q$

$$
\left(\frac{a(Q)}{|Q|}|Q|_{\nu}\right) \mathbb{1}_{Q}(.) \leq C\left(T \mathbb{I}_{Q} \nu\right)(.) \mathbb{1}_{Q}(.) .
$$

B) Let $m \geq 1$. There is $C=C(a, n, m)>0$ so that

$$
\left(T \mathbb{1}_{Q} \nu\right)(.) \mathbb{1}_{m Q}(.) \leq C\left[S_{1}(.)+S_{2}(.)\right]
$$


where

$$
S_{1}(.)=\left(\frac{a(Q)}{|Q|}|Q|_{\nu}\right) \mathbb{1}_{m Q}(.)
$$

and

$$
S_{2}(.)=\frac{a(Q)}{|Q|}\left(\sum_{j \geq 0} 2^{-j n[\lambda-1]} \int_{Q \cap\left\{|y-.| \sim 2^{-j}|Q|^{\frac{1}{n}}\right\}} \nu\right) \mathbb{I}_{m Q}(.)
$$

C) Let $m \geq 4$. There is $C=C(a, n, m)>0$ so that

$$
\left(T \mathbb{I}_{Q} \nu\right)(.) \mathbb{I}_{(m Q)^{c}}(.) \leq C|Q|_{\nu}\left(\sum_{j \geq 0} \frac{a\left(2^{j} Q\right)}{\left|2^{j} Q\right|} \mathbb{I}_{2^{j} Q}\right) .
$$

Proof of Lemma 1:

Let $Q$ be a dyadic cube. Then we have

$$
\int_{Q}(T f \mu) d \omega=\int_{\mathbb{R}^{n}}\left[T^{*} \mathbb{I}_{Q} \omega\right] f d \mu \geq \int_{3 Q}\left[T^{*} \mathbb{I}_{Q} \omega\right] f d \mu
$$

Diving by $|Q|_{\omega}$ this inequality and taking the supremum, we obtain the conclusion.

Proof of Lemma 2:

A) Let $Q$ be a cube with center $x_{0}$ and length $2 R>0$. Then $|x-y| \leq$ $c 2 R$ for all $x, y \in Q$ with $c=c(n)$. We obtain

$$
\begin{aligned}
&\left(\frac{a(Q)}{|Q|}|Q|_{\nu}\right) \mathbb{I}_{Q}(y) \leq c_{1}(a, n)\left(\int_{x \in Q} \frac{a(y, c 2 R)}{R^{n}} d \nu\right) \mathbb{1}_{Q}(y) \\
& \leq c_{2}(a, n)\left[\int_{x \in Q}\left(\frac{|x-y|}{c 2 R}\right)^{n} \frac{1}{|x-y|^{n}} a\right. \\
& \leq c_{3}(a, n)\left[\int_{x \in Q}\left(\frac{|x-y|}{c 2 R}\right)^{n[1-\sigma]} \frac{a(y,|x-y|)}{|x-y|^{n}} d \nu(x)\right] \\
& \mathbb{I}_{Q}(y) .
\end{aligned}
$$

Since $0<\sigma \leq 1$ and $|x-y|<2 c R$ we get

$$
\left(\frac{a(Q)}{|Q|}|Q|_{\nu}\right) \mathbb{I}_{Q}(y) \leq c(a, n)\left(T_{a} \mathbb{I}_{Q} \nu\right)(y) \mathbb{\Perp}_{Q}(y)
$$


B) Let $Q$ be a cube as in part $\mathrm{A}$, and let $m \geq 1$. We can write

$$
\left(T_{a} \mathbb{\Perp}_{Q} \nu\right)(y) \mathbb{1}_{m Q}(y)=S_{1}(y)+S_{2}(y)
$$

where

$$
S_{1}(y)=\left(\int_{Q \cap\{R \leq|y-x|\}} \frac{a(y,|x-y|)}{|x-y|^{n}} d \nu(x)\right) \mathbb{I}_{m Q}(y)
$$

and

$$
S_{2}(y)=\left(\int_{Q \cap\{|y-x|<R\}} \frac{a(y,|x-y|)}{|x-y|^{n}} d \nu(x)\right) \mathbb{I}_{m Q}(y) .
$$

For $S_{1}(y)$ we can observe that for $y \in(m Q)$ and $x \in Q$ then $B(y, \mid x-$ $y \mid) \subset c(n) Q$ and consequently we get

$$
\begin{aligned}
S_{1}(y) & =\left(\int_{Q \cap\{R \leq|y-x|\}} \frac{a(y,|x-y|)}{|x-y|^{n}} d \nu(x)\right) \mathbb{I}_{m Q}(y) \\
& \leq c_{1}(a, n, m) \frac{a(Q)}{|Q|}\left(\int_{Q \cap\{R \leq|y-x|\}} d \nu(x)\right) \mathbb{I}_{m Q}(y) \\
& \leq c_{1}(a, n, m)\left(\frac{a(Q)}{|Q|}|Q|_{\nu}\right) \mathbb{I}_{m Q}(y) .
\end{aligned}
$$

For $S_{2}(y)$ with $R=|Q|^{\frac{1}{n}}$, we have

$$
\begin{aligned}
S_{2}(y) & =\left[\sum_{j \geq 0} \int_{Q \cap\left\{|y-x| \sim 2^{-j} R\right\}} \frac{a(y,|x-y|)}{|x-y|^{n}} d \nu(x)\right] \mathbb{I}_{m Q}(y) \\
& \leq c_{1}(a, n) \sum_{j \geq 0}\left(2^{-j} R\right)^{-n} a\left(y, 2^{-j} R\right)\left(\int_{Q \cap\left\{|y-x| \sim 2^{-j} R\right\}} d \nu(x)\right) \mathbb{I}_{m Q}(y) \\
& \leq c_{2}(a, n, m) \frac{a(Q)}{|Q|}\left[\sum_{j \geq 0} 2^{-j n[\lambda-1]}\left(\int_{Q \cap\left\{|y-x| \sim 2^{-j} R\right\}} d \nu(x)\right)\right] \mathbb{I}_{m Q}(y) .
\end{aligned}
$$

The proof of the part C) lies on the same ideas. We leave the detail for the reader.

\section{Proofs of main results}

Preliminaries for the proof of Theorem 1.

Let $f$ be a nonnegative function bounded and with support compact. By the hypothesis $\left(S_{T}\right)$ then we can observe that

$$
\|T f \mu\|_{L_{\omega}^{q}}<\|f\|_{L^{\infty}}\left\|T \mathbb{1}_{|x|<R} \mu\right\|_{L_{\omega}^{q}}<\infty .
$$


Since $T f \mu$ is semicontinuous, so for each $k \in \mathbb{Z}$ the open set $\Omega_{k}=$ $\left\{T f \mu>2^{k}\right\}$ can be written as $\bigcup_{j} Q_{j k}$ where the $Q_{j k}$ are the dyadic cubes maximal among those dyadic cubes $Q$ satisfying $(R Q) \subset \Omega_{k}$. Choosing $R \geq 3$ sufficiently large (depending only on the dimension $n$ ), we obtain

$$
\Omega_{k}=\bigcup_{j} Q_{j k} \text { where } \operatorname{int}\left(Q_{j k}\right) \cap \operatorname{int}\left(Q_{j^{\prime}}^{k}\right)=\emptyset \text { if } j \neq j^{\prime}
$$

and

$\left(R Q_{j k}\right) \subset \Omega_{k}$ and $\left(3 R Q_{j k}\right) \Omega_{k}^{c} \neq \emptyset$ for all $k, j$ (Whitney condition).

Let fix $m \geq 2$ which we will choose later, and let define $E_{j k}=Q_{j k} \cap$ $\left(\Omega_{k+m-1} \backslash \Omega_{k+m}\right)$. Using the hypothesis $\mathcal{H}$ on the kernel $K$, and the Whitney condition we get

\section{Lemma.}

1) There is $C=C(K, R)>0$ so that for all $k, j$

$$
(T f \mu)(.) \mathbb{I}_{\left(3 Q_{j k}\right)^{c}}(.) \leq C 2^{k} \mathbb{I}_{Q_{j k}}(.) .
$$

2) For a suitable choice of the integer $m$

$$
2^{k} \mathbb{1}_{E_{j k}}(.) \leq\left(T f \mu \mathbb{I}_{Q_{j k}}\right)(.) \mathbb{I}_{E_{j k}}(.)
$$

therefore

$$
\left|E_{j k}\right|_{\omega} \leq 2^{-k} \int_{3 Q_{j k}}\left[T^{*} \omega \mathbb{I}_{Q_{j k}}\right] f d \mu .
$$

By the Whitney condition one can find at least one $z$ which belongs to $\left(3 R Q_{j k}\right) \cap \Omega_{k}^{c}$. It first implies: $(T f \mu)(z) \leq 2^{k}$.

Also for $x \in Q_{j k}$ and $y \in\left(3 Q_{j k}\right)^{c}|z-y| \leq C_{1}|x-y|$ for some $C_{1}=$ $C_{1}(n, R)>0$. So for another constant $C_{2}=C_{2}\left(C_{1}\right)>0: K(x, y) \leq$ $C_{2} K(z, y)$.

The conclusion 1) appears from these two inequalities, indeed we have

$$
\begin{aligned}
\left(T f \mu \mathbb{I}_{\left(3 Q_{j k}\right)^{c}}\right)(x) \mathbb{I}_{Q_{j k}}(x) & =\left(\int_{\left(3 Q_{j k}\right)^{c}} K(x, y) f(y) d \mu(y)\right) \mathbb{I}_{Q_{j k}}(x) \\
& \leq C_{2}\left(\int_{\mathbb{R}^{n}} K(z, y) f(y) d \mu(y)\right) \mathbb{I}_{Q_{j k}}(x) \\
& \leq C_{2}(T f \mu)(z) \mathbb{I}_{Q_{j k}}(x) \leq C_{2} 2^{k} \mathbb{I}_{Q_{j k}}(x) .
\end{aligned}
$$


The part 2) will be a direct consequence of 1). Since $E_{j k}=Q_{j k} \cap$ $\left(\Omega_{k+m-1} \backslash \Omega_{k+m}\right)$ and $\Omega_{k}=\left\{T f \mu>2^{k}\right\}$ then choosing $m \geq 2$ and $2^{m-2} \geq C_{2}$ we get

$$
\begin{aligned}
\left(T f \mu \mathbb{I}_{\left(3 Q_{j k}\right)}\right)(x) \mathbb{I}_{E_{j k}}(x) & =(T f \mu)(x) \mathbb{I}_{E_{j k}}(x)-\left(T f \mu \mathbb{I}_{\left(3 Q_{j k}\right)^{c}}\right)(x) \mathbb{I}_{E_{j k}}(x) \\
& >\left(2^{k+m-1}-2^{k} C_{2}\right)(x) \mathbb{I}_{E_{j k}}(x) \\
& >\left(2^{k+m-1}-2^{k+m-2}\right)(x) \mathbb{I}_{E_{j k}}(x)>2^{k} \mathbb{I}_{E_{j k}}(x) .
\end{aligned}
$$

So, by integration with respect to the measure $d \omega$, this involves

$$
\begin{aligned}
2^{k}\left|E_{j k}\right|_{\omega} & \leq \int_{E_{j k}}\left[T f \mu \mathbb{1}_{3 Q_{j k}}\right] d \omega \\
& \leq \int_{3 Q_{j k}}\left[T^{*} \omega \mathbb{I}_{E_{j k}}\right] f d \mu \leq \int_{3 Q_{j k}}\left[T^{*} \omega \mathbb{I}_{Q_{j k}}\right] f d \mu
\end{aligned}
$$

Proof of the Theorem 1:

Using this Lemma, now we prove the inequality

$$
\|T f \mu\|_{L_{\omega}^{q}} \leq c\left\|M_{T, \omega, \mu}^{d} \mu\right\|_{L_{\omega}^{q}}
$$

Let $\beta \in] 0,1[$ whose value is to be specified later in the course of the proof. Then we get

$$
\begin{aligned}
\|T f \mu\|_{L_{\omega}^{q}}^{q} & \leq c \sum_{k, j} 2^{k q}\left|E_{j k}\right|_{\omega} \quad c=c(q, m) \\
& \left.\leq c \sum_{k, j ;\left|E_{j k}\right|_{\omega} \leq \beta\left|Q_{j k}\right|_{\omega}}+\sum_{k, j ; \beta\left|Q_{j k}\right|_{\omega}<\left|E_{j k}\right|_{\omega}}\right] 2^{k q}\left|E_{j k}\right|_{\omega} \\
& \leq c \beta \sum_{k, j} 2^{k q}\left|Q_{j k}\right|_{\omega}+c \sum_{k, j ; \beta\left|Q_{j k}\right|_{\omega}<\left|E_{j k}\right|_{\omega}} \\
& \left|E_{j k}\right|_{\omega}\left(\frac{1}{\left|E_{j k}\right|_{\omega}} \int_{3 Q_{j k}}\left[T^{*} \omega \mathbb{I}_{Q_{j k}}\right] f d \mu\right)^{q} \\
& \leq c^{\prime} \beta\|T f \mu\|_{L_{\omega}^{q}}^{q}+c \beta^{-q} \sum_{k, j} \\
& \left|E_{j k}\right|_{\omega}\left(\frac{1}{\left|Q_{j k}\right|_{\omega}} \int_{3 Q_{j k}}\left[T^{*} \omega \mathbb{I}_{Q_{j k}}\right] f d \mu\right)^{q} \\
& \leq c^{\prime} \beta\|T f \mu\|_{L_{\omega}^{q}}^{q}+c \beta^{-q} \sum_{k, j} \int_{E_{j k}}\left(M_{T, \omega, \mu}^{d} f \mu\right)^{q}
\end{aligned}
$$




$$
\leq c^{\prime} \beta\|T f \mu\|_{L_{\omega}^{q}}^{q}+c \beta^{-q}\left\|M_{T, \omega, \mu}^{d} f \mu\right\|_{L_{\omega}^{q}}^{q} .
$$

Since $\|T f \mu\|_{L_{\omega}^{q}}^{q}<\infty$, then choosing $\left.\beta \in\right] 0,1\left[\right.$ and $c^{\prime}(q, m) \beta<\frac{1}{2}$, we have

$$
\|T f \mu\|_{L_{\omega}^{q}}^{q} \leq c(q, m)\left\|M_{T, \omega, \mu}^{d} f \mu\right\|_{L_{\omega}^{q}}^{q} .
$$

Therefore the Theorem is proved for each bounded function $f$ with support compact. For a general nonnegative function $f$, we can also obtain the same conclusion by using the monotone convergence theorem.

Proof of proposition 2:

Since the first inequality is proved in Theorem 1 , then we are reduced to get the converse inequality

$$
\left\|M_{T, \omega, \mu}^{d} f \mu\right\|_{L_{\omega}^{q}} \leq C\|T f \mu\|_{L_{\omega}^{q}} .
$$

By Lemma 1: $\left(M_{T, \omega, \mu}^{d} f\right)(.) \leq\left(M_{\omega}^{d} T f \mu\right)($.$) , then the conclusion appears$ if we have

$$
\left\|M_{\omega}^{d} g\right\|_{L_{\omega}^{q}} \leq C\|g\|_{L_{\omega}^{q}} .
$$

By the well known arguments (covering lemma using dyadic cubes and interpolation) then this last maximal inequality is valid for all $q>1$. The same inequality $(*)$ is also true for all $q$ with $0<q \leq 1$ by the means of the extra-hypothesis $M_{\omega}^{d}(T g)(.) \leq c(n, K, \omega)(T g)($.$) .$

Proof of Theorem 3:

Let $f$ be a nonnegative $d \mu$-locally integrable function. Since $\left(T^{*} \nu\right) \approx$ $(T \nu)$ then taking $d \nu=d \omega$ in part A) of Lemma 2, then it appears that for each dyadic cube $Q$

$$
\begin{aligned}
\frac{a(Q)}{|Q|} \int_{Q} f d \mu & =|Q|_{\omega}{ }^{-1} \int_{Q}\left(\frac{a(Q)}{|Q|}|Q|_{\omega}\right) f d \mu \\
& \leq c(a, n)|Q|_{\omega}{ }^{-1} \int_{3 Q}\left(T_{a}^{*} \omega \mathbb{I}_{Q}\right) f d \mu .
\end{aligned}
$$

Hence, we have $\left(M_{\Phi}^{d} f \mu\right) \leq c(a, n)\left(M_{T_{a}, \omega, \mu}^{d} f\right)$.

Conversely in order to get $\left(M_{T_{a}, \omega, \mu}^{d} f \mu\right) \leq C(a, n)\left(M_{\Phi} f \mu\right)$, it is sufficient to get

$$
\left(T_{a}^{*} \mathbb{I}_{Q} \omega\right)(.) \mathbb{I}_{3 Q}(.) \leq C(a, n)\left(\frac{a(c Q)}{|c Q|}|c Q|_{\omega}\right) \mathbb{I}_{c Q}(.)
$$

where $c=c(n) \geq 3$. By part B) of Lemma 3 , the first member of (\$) is essentially dominated by the sum of

$$
S_{1}(.)=\left(\frac{a(Q)}{|Q|}|Q|_{\omega}\right) \mathbb{1}_{3 Q}(.)
$$


and

$$
S_{2}(.)=\frac{a(Q)}{|Q|}\left(\sum_{j \geq 0} 2^{-j n[\lambda-1]} \int_{Q \cap\left\{|y-.| \sim 2^{-j} R\right\}} d \omega(y)\right) \mathbb{I}_{3 Q}(.) .
$$

So it is clear, that it remains to estimate $S_{2}($.$) .$

If $\lambda=1$, then we immediately get

$$
\begin{aligned}
S_{2}(x) & =\frac{a(Q)}{|Q|}\left(\sum_{j \geq 0} \int_{Q \cap\left\{|y-x| \sim 2^{-j} R\right\}} d \omega(y)\right) \mathbb{I}_{3 Q}(x) \\
& \leq c(a, n)\left(\frac{a(Q)}{|Q|}|Q| \omega\right) \mathbb{I}_{3 Q}(x) .
\end{aligned}
$$

Now for $\lambda \in] 0,1\left[\right.$ we use the hypothesis $d \omega R D_{\rho}$ with $1-\lambda<\rho$. We also note that for $x \in(3 Q)$ then $B(x, R) \subset\left(c_{1} Q\right)$ for a constant $c_{1}=c_{1}(n) \geq 3$. Therefore we obtain

$$
\begin{aligned}
S_{2}(x) & \leq \frac{a(Q)}{|Q|}\left(\sum_{j \geq 0} 2^{-j n[\lambda-1]}\left|B\left(x, 2^{-j} R\right)\right|_{\omega}\right) \mathbb{I}_{3 Q}(x) \\
& \leq c(\omega) \frac{a(Q)}{|Q|}\left(\sum_{j \geq 0} 2^{-j n[\lambda-1+\rho]}\right)|B(x, R)|_{\omega} \mathbb{I}_{3 Q}(x) \\
& \leq c^{\prime}(a, \omega) \frac{a(Q)}{|Q|}\left|c_{1} Q\right|_{\omega} \mathbb{I}_{3 Q}(x) \\
& \leq c^{\prime \prime}(a, \omega) \frac{a\left(c_{1} Q\right)}{\left|c_{1} Q\right|}\left|c_{1} Q\right|_{\omega} \mathbb{I}_{c_{1} Q}(x) .
\end{aligned}
$$

Proof of Theorem 5:

It is clear that $(2)$ is a necessary condition for $\left(P_{T}\right)$. To get the condition (1) we first note that for $|x|>R(R>0)$ and $|y|<\frac{1}{2} R$ then $|x-y| \approx|x|$, and consequently taking $f=\mathbb{I}_{B(0, R)}$ in inequality $\left(P_{T}\right)$ we have

$$
\begin{aligned}
\infty>|B(0, R)|_{\mu}^{\frac{1}{p}} & \geq C\left\|\left(\int_{|y|<\frac{1}{2} R} \frac{a(x,|x-y|)}{|x-y|^{n}} d \mu(y)\right) \mathbb{I}_{|x|>R}\right\|_{L_{\omega}^{q}} \\
& \geq C\left|B\left(0, \frac{1}{2} R\right)\right|_{\mu}\left[\int_{|x|>R}\left(\frac{a(x,|x|)}{|x|^{n}}\right)^{q} d \omega(x)\right]^{\frac{1}{q}} .
\end{aligned}
$$

Now we suppose the conditions (1) and (2) are satisfied. The keys for the converse are the following:

$$
\left\|T \mathbb{1}_{B(0, R)} \mu\right\|_{L_{\omega}^{q}}<c(R)<\infty \text { for all } R>0 ;
$$


(ii)

$$
M_{\Phi} \mu: L_{\mu}^{p} \rightarrow L_{\omega}^{q}
$$

Indeed by $(i)$ and $(i i)$ we have

$\|T f \mu\|_{L_{\omega}^{q}} \leq c\left\|M_{T, \omega, \mu} f\right\|_{L_{\omega}^{q}}$ by Theorem 1 and by using (i)

$\leq c\left\|M_{\Phi} f \mu\right\|_{L_{\omega}^{q}}$ by Theorem 3 since $d \omega \in R D_{\rho}$ with $1-\lambda<\rho$

$\leq c\|f\|_{L_{\mu}^{p}}$ by $(i i)$.

To get the point $(i)$, we note that $\left\|\left(T \mathbb{1}_{B(0, R)} \mu\right) \mathbb{1}_{|x|<2 R}\right\|_{L_{\omega}^{q}}<\infty$. On the otherhand, we have

$$
\begin{aligned}
\left\|\left(T \mathbb{I}_{B(0, R)} \mu\right) \mathbb{I}_{|x|>2 R}\right\|_{L_{\omega}^{q}} & \leq c\left\|\left(\int_{|y|<R} \frac{a(x,|x-y|)}{|x-y|^{n}} d \mu(y)\right) \mathbb{I}_{|x|>2 R}\right\|_{L_{\omega}^{q}} \\
& \leq c^{\prime}|B(0, R)|_{\mu}\left[\int_{|x|>2 R}\left(\frac{a(x,|x|)}{|x|^{n}}\right)^{q} d \omega(x)\right]^{\frac{1}{q}}<\infty .
\end{aligned}
$$

By a result in $[\mathbf{R a 1}]$, a sufficient (and necessary) condition for the embedding $(i i)$ is

$$
\left\|\left(M_{\Phi} \sum_{k} \varepsilon_{k} \mathbb{I}_{Q_{k}} \mu\right) \mathbb{I}_{\bigcup Q_{k}}\right\|_{L_{\omega}^{q}} \leq C\left\|\sum_{k} \varepsilon_{k} \mathbb{I}_{Q_{k}} \mu\right\|_{L_{\mu}^{p}}
$$

and $\left\|\left(M_{\Phi} \mathbb{I}_{Q} \mu\right) \mathbb{1}_{Q}\right\|_{L_{\omega}^{q}} \leq C\left\|\mathbb{1}_{Q}\right\|_{L_{\mu}^{p}}$ if $p \leq q$. By Lemma $\left.2 \mathrm{~A}\right)$ then $\left(M_{\Phi} f \mu\right) \leq c(T f \mu)$ and consequently the condition (2) in Theorem 5 implies the above one.

Proof of Proposition 6:

To prove the first part of Proposition 6, we suppose

$$
\left\|\left(T \mathbb{I}_{Q} \mu\right) \mathbb{1}_{Q}\right\|_{L_{\omega}^{q}} \leq A\left\|\mathbb{1}_{Q}\right\|_{L_{\mu}^{p}} \text { for all cubes } Q .
$$

Since $1<p \leq q$ this condition implies $M_{\Phi} \mu: L_{\mu}^{p} \rightarrow L_{\omega}^{q}$. And as above to get $(i)$ it is sufficient to prove

$$
\left\|\left(T \mathbb{I}_{Q} \mu\right) \mathbb{I}_{(m Q)^{c}}\right\|_{L_{\omega}^{q}} \leq C\left\|\mathbb{I}_{Q}\right\|_{L_{\mu}^{p}}
$$

with a constant $m \geq 4$. Using the fact that $d \mu \in R D_{\rho^{\prime}}$ for some $\rho^{\prime}>0$, then by Lemma 2 (part C) we get

$$
\left\|\left(T \mathbb{1}_{Q} \mu\right) \mathbb{1}_{(m Q)^{c}}\right\|_{L_{\omega}^{q}} \leq c_{1}|Q|_{\mu} \sum_{j \geq 0} \frac{a\left(2^{j} Q\right)}{\left|2^{j} Q\right|}\left|2^{j} Q\right|_{\omega}^{\frac{1}{q}}
$$




$$
\begin{aligned}
& \leq c_{2}|Q|_{\mu} \sum_{j \geq 0}\left|2^{j} Q\right|_{\mu}^{-1}\left\|\left(T \mathbb{I}_{2^{j} Q} \mu\right) \mathbb{I}_{\left(2^{j} Q\right)}\right\|_{L_{\omega}^{q}} \\
& \leq c_{2} A|Q|^{\frac{1}{p}} \sum_{j \geq 0}\left(\frac{|Q|_{\mu}}{\left|2^{j} Q\right|_{\mu}}\right)^{1-\frac{1}{p}} \\
& \leq c_{3} A|Q|_{\mu}^{\frac{1}{p}} .
\end{aligned}
$$

For the second part of this proposition, the point is to note that $M_{\Phi} \mu$ : $L_{\mu}^{p} \rightarrow L_{\omega}^{q}$ is equivalent to

$$
\frac{a(Q)}{|Q|}|Q|_{\mu}^{1-\frac{1}{p}}|Q|_{\omega}^{\frac{1}{q}}<A<\infty
$$

whenever $d \mu \in A_{\infty}$ (see $[\mathbf{P e}]$ ) or $d \mu \in R D_{\infty^{\prime}}$ with $1-\lambda<\rho^{\prime}$ (see $[\operatorname{Ra} 2])$.

Proof of Proposition 7:

It is clear that a necessary condition for $\left(P_{T}\right)$ is

$$
\left\|\sum_{k} \varepsilon_{k}\left(T \mathbb{1}_{Q_{k}} \mu\right) \mathbb{I}_{\left(m Q_{k}\right)}\right\|_{L_{\omega}^{q}} \leq A\left\|\sum_{k} \varepsilon_{k} \mathbb{I}_{Q_{k}}\right\|_{L_{\mu}^{p}}
$$

with $m \geq 4$ and for all cubes $Q, Q_{k}$ and all $\varepsilon_{k}>0$. Conversely we suppose this condition be satisfied and $d \mu \in \widetilde{R D}(p)$. Once we have

$$
\left\|\sum_{k} \varepsilon_{k}\left(T \mathbb{1}_{Q_{k}} \mu\right) \mathbb{I}_{\left(m Q_{k}\right)^{c}}\right\|_{L_{\omega}^{q}} \leq c A\left\|\sum_{k} \varepsilon_{k} \mathbb{I}_{Q_{k}}\right\|_{L_{\mu}^{p}}
$$

then $(i)$ and $(i i)$ hold as in proof of Theorem 5, and consequently the inequality $\left(P_{T}\right)$ is satisfied. Now using Part C) of Lemma 2, the above condition $(*)$ and the hypothesis $d \omega \in \widetilde{R D}(p)$ we have

$$
\begin{aligned}
\mathcal{S} & =\left\|\sum_{k} \varepsilon_{k}\left(T \mathbb{I}_{Q_{k}} \mu\right) \mathbb{I}_{\left(m Q_{k}\right)^{c}}\right\|_{L_{\omega}^{q}} \\
& \leq c_{1}\left\|\sum_{k} \varepsilon_{k} \sum_{j \geq 0} \frac{a\left(2^{j} Q_{k}\right)}{\left|2^{j} Q_{k}\right|}\left|Q_{k}\right|_{\mu} \mathbb{I}_{\left(2^{j} Q_{k}\right)}\right\|_{L_{\omega}^{q}} \text { by part C of Lemma } 2 \\
& \leq c_{2}\left\|\sum_{k} \varepsilon_{k} \sum_{j \geq 0}\left(\frac{\left|Q_{k}\right|_{\mu}}{\left|2^{j} Q_{k}\right|_{\mu}}\right)\left(T \mathbb{1}_{2^{j} Q_{k}} \mu\right) \mathbb{I}_{\left(2^{j} Q_{k}\right)}\right\|_{L_{\omega}^{q}}
\end{aligned}
$$




$$
\begin{aligned}
& \leq c_{2} A\left\|\sum_{j \geq 0} \sum_{k} \varepsilon_{k}\left(\frac{\left|Q_{k}\right|_{\mu}}{\left|2^{j} Q_{k}\right|_{\mu}}\right) \mathbb{I}_{\left(2^{j} Q_{k}\right)}\right\|_{L_{\mu}^{p}} \text { by the condition }(*) \\
& \leq c_{3} A\left\|\sum_{k} \varepsilon_{k} \mathbb{I}_{Q_{k}}\right\|_{L_{\mu}^{p}} \text { since } d \mu \in \widetilde{R D}(p) .
\end{aligned}
$$

It is also clear that a necessary condition for $\left(P_{T}\right)$ is

$$
\left\|\sum_{k} \varepsilon_{k}\left(\frac{a\left(Q_{k}\right)}{\left|Q_{k}\right|}\left|Q_{k}\right|_{\mu}\right) \mathbb{1}_{Q_{k}}\right\|_{L_{\omega}^{q}} \leq A\left\|\sum_{k} \varepsilon_{k} \mathbb{I}_{Q_{k}}\right\|_{L_{\mu}^{p}} .
$$

Conversely we assume this condition be satified and $d \mu \in D_{\varepsilon^{\prime}, p} \cap R D_{\rho^{\prime}}$ with $1-\lambda<\rho^{\prime}$ and $\varepsilon^{\prime}<p \rho^{\prime}$. It is sufficient to get the conditions in the first part of the present Proposition. As in the proof of Theorem 3 by using part A) of Lemma 2) and since $d \mu \in D_{\infty}$ then

$$
\left(T \mathbb{1}_{Q_{k}} \mu\right) \mathbb{1}_{\left(m Q_{k}\right)} \leq c\left(\frac{a\left(Q_{k}\right)}{\left|Q_{k}\right|}\left|Q_{k}\right|_{\mu}\right) \mathbb{1}_{\left(m Q_{k}\right)}
$$

and consequently

$$
\begin{aligned}
\left\|\sum_{k} \varepsilon_{k}\left(T \mathbb{I}_{Q_{k}} \mu\right) \mathbb{I}_{\left(m Q_{k}\right)}\right\|_{L_{\omega}^{q}} & \leq c\left\|\sum_{k} \varepsilon_{k}\left(\frac{a\left(Q_{k}\right)}{\left|Q_{k}\right|}\left|Q_{k}\right| \mu\right) \mathbb{I}_{\left(m Q_{k}\right)}\right\|_{L_{\omega}^{q}} \\
& \leq c\left\|\sum_{k} \varepsilon_{k} \mathbb{I}_{\left(m Q_{k}\right)}\right\|_{L_{\mu}^{p}} .
\end{aligned}
$$

Now using $d \mu \in D_{\varepsilon^{\prime}, p} \cap R D_{\rho^{\prime}}$ with $\varepsilon^{\prime}<p \rho^{\prime}$ we can get the condition $d \mu \in \widetilde{R D}(p)$ as follow:

$$
\begin{aligned}
\mathcal{S} & =\left\|\sum_{k} \varepsilon_{k} \sum_{j \geq 0}\left(\frac{\left|Q_{k}\right|_{\mu}}{\left|2^{j} Q_{k}\right|_{\mu}}\left|Q_{k}\right|_{\mu}\right) \mathbb{I}_{\left(2^{j} Q_{k}\right)}\right\|_{L_{\mu}^{p}} \\
& \leq c_{1} \sum_{j \geq 0} 2^{-j n \rho^{\prime}}\left\|\sum_{k} \varepsilon_{k} \mathbb{I}_{\left(2^{j} Q_{k}\right)}\right\|_{L_{\mu}^{p}} \\
& \leq c_{2} \sum_{j \geq 0} 2^{-j n\left[\rho^{\prime}-\frac{1}{p} \varepsilon^{\prime}\right]}\left\|\sum_{k} \varepsilon_{k} \mathbb{I}_{Q_{k}}\right\|_{L_{\mu}^{p}}=c_{3}\left\|\sum_{k} \varepsilon_{k} \mathbb{I}_{Q_{k}}\right\|_{L_{\mu}^{p}} .
\end{aligned}
$$

Finally we suppose $d \mu \in D_{\infty} \cap R D_{\rho^{\prime}}$ and $d \omega \in D_{\varepsilon, q} \cap R D_{\rho}$ with $1-\lambda<\rho^{\prime}$ and $\varepsilon<q(1-\sigma)$. It remains to get the above condition (**). Thus we have

$$
\mathcal{S}=\left\|\sum_{k} \varepsilon_{k}\left(T \mathbb{1}_{Q_{k}} \mu\right) \mathbb{1}_{\left(m Q_{k}\right)^{c}}\right\|_{L_{\omega}^{q}}
$$




$$
\begin{aligned}
& \leq c_{1}\left\|\sum_{k} \varepsilon_{k} \sum_{j \geq 0}\left(\frac{a\left(2^{j} Q_{k}\right)}{\left|2^{j} Q_{k}\right|}\left|Q_{k}\right|_{\mu}\right) \mathbb{I}_{\left(2^{j} Q_{k}\right)}\right\|_{L_{\omega}^{q}}\left\|\sum_{j \geq 0} 2^{-j n[1-\sigma]}\right\| \sum_{k} \varepsilon_{k}\left(\frac{a\left(Q_{k}\right)}{\left|Q_{k}\right|}\left|Q_{k}\right|_{\mu}\right) \mathbb{I}_{\left(2^{j} Q_{k}\right)} \|_{L_{\omega}^{q}} \\
& \leq c_{3} \sum_{j \geq 0} 2^{-j n\left[1-\sigma-\frac{1}{q} \varepsilon\right]}\left\|\sum_{k} \varepsilon_{k}\left(\frac{a\left(Q_{k}\right)}{\left|Q_{k}\right|}\left|Q_{k}\right|_{\mu}\right) \mathbb{I}_{Q_{k}}\right\|_{L_{\omega}^{q}} \\
& \leq c_{3} A \sum_{j \geq 0} 2^{-j n\left[1-\sigma-\frac{1}{q} \varepsilon\right]}\left\|\sum_{k} \varepsilon_{k} \mathbb{I}_{Q_{k}}\right\|_{L_{\mu}^{p}} \\
& \leq c_{4} A\left\|\sum_{k} \varepsilon_{k} \mathbb{I}_{Q_{k}}\right\| \|_{L_{\mu}^{p}} .
\end{aligned}
$$

Acknowledgement. I would like to thank the referee for his helpful comments and suggestions.

\section{References}

[Ad] D. AdAms, A note on Riesz potentials, Duke Math. J. 42 (1975), 765-778.

[Fr-Ja] M. Frazier AND B. JAWERTh, A discrete transform and decomposition of distribution spaces, J. Funct. Anal. 93(1) (1990), 34-170.

[Ke-Sa] R. Kerman And E. SAWyer, Weighted norm inequalities for potentials with applications to Schrodinger operators, Fourier transforms and Carleson measures, Ann. Inst. Fourier 36 (1986), 207-228.

[Mu-Wh] B. Muckenhoupt And R. L. WheEden, Weighted norm inequalities for fractional integrals, Trans. Amer. Math. Soc. 192 (1974), 261-274.

[Pe] C. PÉrEz, Weighted norm inequalities for potential and maximal operators, Ph. D. Thesis, Washington University (1989).

[Ra1] Y. RAKOTONDRATsimbA, Inégalités à poids pour des opérateurs maximaux et des opérateurs de type potentiel., Thèse de Doctorat, Université d'Orléans France (1991).

[Ra2] Y. Rakotondratsimba, On Muckenhoupt and Sawyer conditions for maximal operators, Pub. Mat. 37 (1993), 57-93. 
[Sa] E. SAwYER, A characterization of a two weight norm inequality for maximal operators, Studia Math. 75 (1982), 1-11.

[Sa-Wh] E. SAWYER AND R. L. WheEden, Weighted norm inequalities for fractional integral on euclidean and homogeneous spaces, Amer. J. Math. 114 (1992), 813-874.

[Ya] K. Yabuta, Sharp maximal functions and $C_{p}$ condition, Archiv. Math. 55 (1990), 151-155.

Keywords. Weighted inequalities, Potential operators, Maximal operators 1980 Mathematics subject classifications: 42B25

\author{
Université d'Orléans \\ Département de Mathématiques \\ U.F.R. Faculté des Sciences \\ B.P. 6759 \\ 45067 Orléans Cedex 2 \\ FRANCE
}

Primera versió rebuda el 16 de Març de 1993,

darrera versió rebuda el 15 de Gener de 1995 